\title{
Synthesis Control in the Systems with Distributed and Concentrated Parameters

\author{
$\square^{1}$ Bulat Faritovich Bairamov, ${ }^{2}$ Farit Davletovich Bairamov \\ ${ }^{1,2}$ Department of Mechanics and Design, Automotive Department, Naberezhnye Chelny Institute (Branch), KFU \\ bbairamov@gmail.com \\ FDBajramov@kpfu.ru
}

Received: 21st August 2020, Accepted: 14th September 2020, Published: 31st October 2020

\begin{abstract}
They consider a controlled system with distributed and lumped parameters, the perturbed state of which is described by linear differential equations in partial and ordinary derivatives. The system consists of one distributed link with finite-dimensional links at its both ends (for example, the systems containing electrical circuits, sufficiently long elastic shafts, the pipelines in which it is necessary to take into account the distributed nature of the fluid flow, etc.). The paper studies the problem of a lumped optimal control development applied to finite-dimensional links and/or to the end of a distributed link that ensures the stable system operation. Two tasks are solved to develop such control. First, the method of Lyapunov functions is used to determine the set of controls that ensure the asymptotic stability of the closed-loop system; then, the Lagrange multiplier method is used on this set to develop an optimal control with the smallest value of the norm at each moment of time. At that, the original equations in private higher-order partial differential equations are represented as a system of evolutionary equations and first-order partial differential equations by introducing additional variables. The constraint equations are the equations without time derivatives. A modified method of Lagrange multipliers is used to take such equations into account when calculating the derivative of the Lyapunov function in view of the system under consideration. The transition to partial differential equations of the first order, together with the development of ordinary differential equations in the standard Cauchy form, allows to construct the Lyapunov function as the sum of integral and ordinary quadratic forms constructively using specific equations, the sign-definiteness of which is checked by the Sylvester criterion, to ensure the stability of the closedloop system and obtain simpler control laws as the linear functions of phase coordinates. In the case of onedimensional distributed systems, these controls can be quite simply implemented in the form of lumped boundary controls that require measuring the system state only at the boundary points, which is of great practical importance. The results obtained in this work significantly expand the possibilities of Lyapunov functions method use when they solve applied problems of control synthesis in engineering objects with distributed and lumped parameters. As an example, we consider the problem of determining the minimum boost pressure of the fuel tank, which ensures stable operation of the heating furnace.
\end{abstract}

\section{Keywords}

The System With Distributed And Lumped Parameters; Control Synthesis; The Method Of Lyapunov Functions; Quadratic Forms.

\section{Introduction}

The method of Lyapunov functions is widely used to study the stability and solve the problems of control synthesis in the systems with distributed parameters. There are fairly complete reviews of the problems in this area [8; $10 ; 4]$. Along with theoretical studies by the method of Lyapunov functions, they performed the studies of specific objects with distributed parameters, for example, elastic and aeroelastic objects $[4 ; 7 ; 9 ; 1 ; 2 ; 3]$, liquid-propellant rocket engines $[8 ; 10 ; 4]$, and others $[8 ; 4 ; 5]$. In applications, the main difficulty is the development of the corresponding Lyapunov functionals, which were usually constructed intuitively during the study of specific objects, proceeding from the total energy, first integrals, and other considerations. In the problems of control synthesis within the systems with distributed parameters, Lyapunov functions are often constructed in the form of double integral forms. Such forms are quite universal, but at present, there are no methods for their sign-definiteness check. This makes it challenging to ensure closed system stability. In this regard, when they solve the problems of stability and synthesis of controls in the systems with distributed and lumped parameters, it is proposed to transform the initial high-order differential equations into a system of first-order equations in time and spatial coordinates and construct the Lyapunov functions as the sum of ordinary and single integral forms, whose sign-definiteness can be checked by Sylvester's criterion application. This approach significantly expands the possibilities of Lyapunov functions method use when they solve the problems of stability and synthesis of controls in engineering systems with distributed and lumped 
parameters.

\section{Methods}

The work uses mathematically rigorous and accurate research methods: the methods of higher-order differential equation transformation into a system of first-order equations, the method of Lyapunov functions, the process of Lagrange multipliers, and matrix theory.

During the calculation of the Lyapunov function derivative (2.1) by virtue of equations (1.1) - (1.3), to take into account the second equations (1.1), (1.3) without time derivatives, an approach is used, which is similar in its procedure to the Lagrange multiplier method.

The stability conditions are written based on well-known classical results from the theory of stability of finitedimensional and distributed systems. The Sylvester criterion checks the sign-definiteness of standard and integral quadratic forms.

\section{Results and Discussion}

1. Problem statement. Let's consider a control system with one distributed and other finite-dimensional links, the perturbed state of which is described by the following equations:

$$
\begin{aligned}
& \frac{\partial \varphi}{\partial t}=A(x) \frac{\partial \varphi}{\partial x}+B(x) \frac{\partial \psi}{\partial x}+A_{0}(x) \varphi+B_{0}(x) \psi \\
& C(x) \frac{\partial \varphi}{\partial x}+D(x) \frac{\partial \psi}{\partial x}+C_{0}(x) \varphi+D_{0}(x) \psi=0 \\
& x \in(0,1) \\
& \frac{d z}{d t}=F_{1} z+F_{2} \varphi(0, t)+F_{3} \varphi(1, t)+G u_{1} \\
& t \in I=(0, \infty)
\end{aligned}
$$

Where $\varphi=\varphi(x, t)-n-$ vector of the phase functions of the distributed link, $\psi=\psi(x, t)-\ell-$ the dimensional vector of the phase functions of this link, the time derivatives of which are not included in the system (1.1), $z=z(t)-m-$ the dimensional vector of the phase functions of the finite-dimensional links, $u_{1}=u_{1}(t)$ $-r-$ the dimensional control vector applied to the finite-dimensional links, $u_{1} \in L_{2}(I), A(x), B(x)$, $C(x), D(x), A_{0}(x), B_{0}(x), C_{0}(x), D_{0}(x)$ - the matrices whose elements are bounded continuous functions, $F_{1}, F_{2}, F_{3}, G$ - the matrices of constants, $L_{2}(I)$ - the space of functions summable with a square at $t \in I$.

Let the values of the first $n_{1} \leq n$ of the functions $\varphi_{i}$ at $x=0$ are used as boundary controls, and the following boundary conditions are given:

$$
\Gamma_{0} \varphi(0, t)=u_{2}, \quad \Gamma_{1} \varphi(1, t)=\Gamma_{2} z
$$

where $\Gamma_{0}-n_{1} \times n-$ the matrix with the elements $\delta_{i i}=1, \delta_{i j}(i \neq j), \Gamma_{1}, \Gamma_{2}-$ the matrices of constants, $u_{2}-n_{1}-$ dimensional vector of boundary control, $u_{2} \in L_{2}(I)$.

Let's denote $u=\left(u_{1}, u_{2}\right)^{T}\left(T-\right.$ the transposition symbol). Any control $u \in L_{2}(I)$ is considered valid.

From the mathematical point of view, the problem (1.1) - (1.3) is a boundary value problem for partial differential equations with complex boundary conditions. The equations (1.1) represent the general form of any linear partial differential equation of arbitrary order in the form of the first order partial differential equations [4; 1]. To transform higher-order equations to the form (1.1), one should take the lowest derivatives as additional variables and write down the original equation and the integrability condition in these variables. 
The equations (1.3) are simple boundary conditions containing the control $u_{2}$. The equation of dynamics (1.2) of finite-dimensional links located at both ends of the distributed link contains boundary values $\varphi(x, t)$ and represents a complex boundary condition in the form of a differential equation.

The equations of the type (1.1) - (1.3) describe the systems that have transmission shafts of considerable length, for example, between the engine and the working machine (generator, pump, compressor, etc.); the systems containing electrical circuits or pipelines (lines), in which it is necessary to take into account the distributed nature of liquid or gas flow, etc.

The following problem is posed. The following is required:

a) Select the set of $\Omega$ controls $u=\left(u_{1}, u_{2}\right)^{T}$ inadmissible controls, that ensure the asymptotic stability of the system (1.1) - (1.3) with respect to the variables $z, \rho=\int_{0}^{1} \varphi^{T} \varphi d x$, where $\rho$ - the measure characterizing the disturbed state of the distributed link.

b) Find the optimal control $u_{O}=\left(u_{1 O}, u_{2 O}\right)^{T}$ by the set of $\Omega$ with the smallest value of the norm square, i.e. the measure $\rho_{u}=\|u\|^{2}=\left\|u_{1}\right\|^{2}+\left\|u_{2}\right\|^{2}$ at each moment.

1. Solution of the problem a. Let's consider the Lyapunov function

$$
V=V_{1}+V_{2}=\int_{0}^{1} \varphi^{T}(x, t) v(x) \varphi(x, t) d x+z^{T}(t) Q z(t),
$$

where $v(x), Q$ - symmetric matrices: the elements $Q$ are constants, and the elements $v(x)$ are continuous bounded functions.

A feature of the system (1.1) - (1.3) is that the second equation (1.1) and the equations (1.3) do not contain time $t$ derivatives. This does not allow to calculate the derivative $V$ directly due to the entire system. First, we calculate the derivative $d V / d t$ due to the first equation in (1.1) and the equation (1.2):

$$
\begin{aligned}
& \frac{d V}{d t}=\int_{0}^{1}\left[\varphi^{T} v\left(A \frac{\partial \varphi}{\partial x}+B \frac{\partial \psi}{\partial x}\right)+\left(\frac{\partial \varphi^{T}}{\partial x} A^{T}+\frac{\partial \psi^{T}}{\partial x} B^{T}\right) v \varphi+\right. \\
& \left.+\varphi^{T}\left(v A_{0}+A_{0}^{T} v\right) \varphi+\varphi^{T} v B_{0} \psi+\psi^{T} B_{0}^{T} v \varphi\right] d x+ \\
& +z^{T}\left(Q F_{1}+F_{1}^{T} Q\right) z+2 \varphi^{T}(0, t) F_{2}^{T} Q z+2 \varphi^{T}(1, t) F_{3}^{T} Q z+2 z^{T} Q G u_{1} .
\end{aligned}
$$

Following the Lagrange multiplier method, we add to this derivative the following expression to take into account the second equations (1.1) and (1.3):

$$
\begin{aligned}
& \int_{0}^{1}\left[\left(\varphi^{T} P_{1}+\psi^{T} P_{2}\right)\left(C \frac{\partial \varphi}{\partial x}+D \frac{\partial \psi}{\partial x}+C_{0} \varphi+D_{0} \psi\right)+\right. \\
& \left.+\left(\frac{\partial \varphi^{T}}{\partial x} C^{T}+\frac{\partial \psi^{T}}{\partial x} D^{T}+\varphi^{T} C_{0}^{T}+\psi^{T} D_{0}^{T}\right)\left(P_{1}^{T} \varphi+P_{2}^{T} \psi\right)\right] d x=0, \\
& {\left[\varphi^{T}(1, t) R_{1}+z^{T} R_{2}\right]\left[\Gamma_{1} \varphi(1, t)-\Gamma_{2} z\right]+\left[\varphi^{T}(1, t) \Gamma_{1}^{T}-z^{T} \Gamma_{2}^{T}\right] \times} \\
& \times\left[R_{1}^{T} \varphi(1, t)+R_{2}^{T} z\right]=0,
\end{aligned}
$$


where $P_{1}=P_{1}(x), P_{2}=P_{2}(x)$, while $R_{1}, R_{2}$ are arbitrary matrices: $P_{1}, P_{2}$ - continuous, $R_{1}, R_{2}-$ constant. The brackets $\left(\varphi^{T} P_{1}+\psi^{T} P_{2}\right),\left(P_{1}^{T} \varphi+P_{2}^{T} \psi\right),\left[\varphi^{T}(1, t) R_{1}+z^{T} R_{2}\right]$, $\left[R_{1}^{T} \varphi(1, t)+R_{2}^{T} z\right]$ play the role of Lagrange multipliers.

Let us perform integration by parts and require that the matrices $v, P_{1}, P_{2}, Q, R_{1}, R_{2}$ satisfy the following conditions:

$$
\begin{aligned}
& v A+P_{1} C=A^{T} v+C^{T} P_{1}^{T}, \quad P_{2} D=D^{T} P_{2}^{T}, \\
& v B+P_{1} D=C^{T} P_{2}^{T}, \quad P_{2} D_{0}+D_{0}^{T} P_{2}^{T}=\frac{d P_{2} D}{d x}, \\
& v B_{0}+P_{1} D_{0}+C_{0}^{T} P_{2}^{T}=\frac{d\left(v B+P_{1} D\right)}{d x}, \quad x \in(0,1) . \\
& v(1) A(1)+P_{1}(1) C(1)+R_{1} \Gamma_{1}+\Gamma_{1}^{T} R_{1}^{T}=0, \\
& Q F_{3}+R_{2} \Gamma_{1}-\Gamma_{2}^{T} R_{1}^{T}=0, \\
& \left.\left(P_{2} D\right)\right|_{0} ^{1}=\left.\left(v B+P_{1} D\right)\right|_{0} ^{1}=0 .
\end{aligned}
$$

Then, taking into account the first equation (1.3), we obtain the following:

$$
\begin{aligned}
& \frac{d V}{d t}=\int_{0}^{1} \varphi^{T} N \varphi d x+z^{T} M z+2 z^{T} Q G u_{1}+u_{2}^{T} Q_{1} u_{2}+\theta(0, t) Q_{2} \theta(0, t)+ \\
& +2 u_{2}^{T} Q_{3} \theta(0, t),
\end{aligned}
$$

Where $\quad N=-\frac{d\left(v A+P_{1} C\right)}{d x}+v A_{0}+A_{0}^{T} v+P_{1} C_{0}+C_{0}^{T} P_{1}^{T}, \quad M=Q F_{1}+F_{1}^{T} Q+R_{2} \Gamma_{2}+\Gamma_{2}^{T} R_{2}^{T}$, $\theta=\left(\varphi_{n_{1}+1}, \ldots, \varphi_{n}\right)^{T}, Q_{j}(j=\overline{1,3})-n_{1} \times n_{1},\left(n-n_{1}\right) \times\left(n-n_{1}\right), n_{1} \times\left(n-n_{1}\right)-$ the matrices that divide the matrix $-\left[v(0) A(0)+P_{1}(0) C(0)\right]$ into blocks as follows: $\left[\begin{array}{c:c}Q_{1} & Q_{3} \\ \hdashline Q_{3}^{T} & Q_{2}\end{array}\right]$.

Let us introduce the functional $W=\int_{0}^{1} \varphi^{T}(x, t) w(x) \varphi(x, t) d x+z^{T} \omega z$, where $w(x), \omega$ are symmetric matrices; $\omega$ - constant, and the elements $w(x)$ are continuous bounded functions.

Within the framework of the method of Lyapunov functions, the solution of the problem a gives the following statement 1: any control $u=\left(u_{1}, u_{2}\right)^{T} \in \Omega$, ensuring the fulfilment of the inequality

$$
\frac{d V}{d t} \leq-W
$$

where $d V / d t$ is determined by the expression (2.4), guarantees the asymptotic stability of the solution $\varphi=\psi=z=0$ of the system (1.1) - (1.3) by variables $z, \rho$, если интегральная квадратичная форма $V_{1}$ (2.1) if the integral quadratic form (2.1) is continuous and definitely positive in the measure $\rho$, the quadratic form 
$V_{2}$ (2.1) is definitely positive, and the derivative $d V / d t$ (2.4) is definitely negative in variables $z, \rho$ [8;6].

The continuity of the integral form $V_{1}$ in measure $\rho$ directly follows from the boundedness of the matrix elements $v(x)$. The remaining conditions of this statement will be satisfied if the matrices $Q$ and $\omega$ are definitely positive, and $v(x)$ and $w(x)$ are definitely positive at $x \in[0,1]$, т.e.

$$
Q>0, \quad \omega>0 ; \quad v(x)>0, \quad w(x)>0, \quad x \in[0,1] .
$$

2. Solution of the problem b. Let's denote the set of controls via $\Omega_{1} \subset \Omega$ satisfying the following equality:

$$
\frac{d V}{d t}=-W
$$

and, therefore, ensuring the asymptotic stability of the solution $\varphi=\psi=z=0$ by the variables $z, \rho$.

On the set of $\Omega_{1}$ let's find the optimal control $u_{O}=\left(u_{1 O}, u_{2 O}\right)^{T}$, that minimizes the value $\rho_{u}$ at each moment of time $t \in I$.

Let's compose the functional $L=u^{T} u+\lambda(d V / d t+W)$, where $\lambda$ is the Lagrange multiplier. From the condition of reaching $\min L$ by $u$, i.e. from the conditions $\delta L=0$ и $\delta^{2} L>0$, we find the optimal control

$$
\begin{aligned}
& u_{1 O}=-\lambda G^{T} Q z, \\
& u_{2 O}=-\lambda\left(E+\lambda Q_{1}\right)^{-1} Q_{3} \theta(0, t), \quad E+\lambda Q_{1}>0,
\end{aligned}
$$

where $E$ is the identity matrix. Substituting these controls into (2.4) from the equality (3.1), we obtain the following equations:

$$
\begin{aligned}
& N+w=0, \quad x \in[0,1], \\
& M-\lambda Q G G^{T} Q+\omega=0, \\
& Q_{2}-\lambda Q_{3}^{T}\left(E+\lambda Q_{1}\right)^{-1}\left(2 E+\lambda Q_{1}\right)\left(E+\lambda Q_{1}\right)^{-1} Q_{3}=0,
\end{aligned}
$$

which together with (2.2), (2.3) are used to develop the matrices $v, Q$. The matrices $P_{1}, P_{2}, R_{1}, R_{2}$ are also determined from the equations (2.2), (2.3).

The value $\lambda$, entering into the controls (3.2) and the equations (3.3) must satisfy the condition $\lambda>0$. If $\lambda \leq 0$, then we obtain $(d V / d t)_{u=0} \leq(d V / d t)_{u=u_{0}}$ from (2.4) taking into account (3.2), i.e. at $\lambda \leq 0$ the control $u_{0}$ does not help to ensure the asymptotic stability of the solution $\varphi=\psi=z=0$.

Let us show that if $\lambda>0$, then the control $u_{0}$ (3.2) minimizes the value $\rho_{u}$ on the set of controls that ensure the fulfilment of not only the equality (3.1), but also the inequality (2.5). For this control, we represent in the form $u_{1}=u_{1 O}+\Delta u_{1}, u_{2}=u_{2 O}+\Delta u_{2}$. Substituting them into (2.4) and using (3.2), (3.3), we find that the inequality (2.5) is satisfied if

$$
2\left(\Delta u_{1}^{T} u_{1 O}+\Delta u_{2}^{T} u_{2 O}\right) \geq \lambda \Delta u_{2}^{T} Q_{1} \Delta u_{2}
$$

Now, taking into account the inequalities (3.4) and (3.2), we obtain the following by direct calculation:

$$
\begin{aligned}
& \Delta \rho_{u}=u^{T} u-u_{O}^{T} u_{O}=\Delta u^{T} \Delta u+2\left(\Delta u_{1} u_{1 O}+\Delta u_{2} u_{2 O}\right) \geq \\
& \geq \Delta u_{2}^{T} \Delta u_{2}+\Delta u_{2}^{T}\left(E+\lambda Q_{1}\right) \Delta u_{2}>0,
\end{aligned}
$$

i.e. indeed, $u_{0}$ minimizes the quantity of $\rho_{u}$ on the set of controls that ensure the inequality (2.5). 
Thus, the following statement 2 holds: the control $u_{0}$ (3.2) is the solution to the problem $\mathrm{b}$ if $\lambda>0$ and the conditions (2.6) are satisfied.

3. Example. Let's consider the system consisting of a heating furnace and a pipeline for fuel supply from the tank to the furnace. The pipeline has a considerable length, and it is necessary to take into account the distributed nature of the flow parameters. The fuel flow in the pipeline is described by the equations [1].

$$
\frac{\partial \varphi_{1}}{\partial t}=-b \frac{\partial \varphi_{2}}{\partial x}, \quad \frac{\partial \varphi_{2}}{\partial t}=-d \frac{\partial \varphi_{1}}{\partial x}, \quad x \in(0,1)
$$

where $x=\frac{y}{\ell}, \varphi_{1}(x, t)=\frac{P(x, t)-P_{*}}{P_{*}}, \varphi_{2}(x, t)=\frac{g(x, t)-g_{*}}{g_{*}}, b=\frac{a^{2} g_{*}}{P_{*} F \ell}, d=\frac{P_{*} F}{g_{*} \ell}, P(x, t)$, $g(x, t)$ - the pressure and fuel consumption in the pipeline, $y$ - the coordinate of the pipeline cross-sections, measured from the tank, $a$ - the speed of sound in the liquid, $F, \ell$ - the cross-sectional area and the pipeline length, the sign $(*)$ indicates the values of the quantities in the design operating mode of the system.

The boundary conditions at $x=0$ and $x=1$ :

$$
\varphi_{1}(0, t)=u_{2}, \quad \varphi_{2}(1, t)=g
$$

where $g$ is the fuel consumption in the furnace, $u_{2}$ - the relative pressure in the tank created by the pressurization system. We will take this pressure for control.

The change of temperature in the furnace can be described by the equation of the inertial link:

$$
\theta \frac{d z}{d t}+z=k_{1} g
$$

where $z=\frac{T-T_{*}}{T_{*}}, T-$ the furnace temperature, $\theta-$ the furnace time constant, $k_{1}>0-$ the constant gain.

Introducing the vector $\varphi=\left(\varphi_{1}, \varphi_{2}\right)^{T}$ let's put down the equations (4.1) - (4.3) in the form of the system (1.1)(1.3), where $F_{1}=-\frac{1}{\theta}, F_{3}=\frac{k_{1}}{\theta}, \Gamma_{0}=\Gamma_{1}=\Gamma_{2}=1, A=\left\|\begin{array}{cc}a & -b \\ -d & 0\end{array}\right\|$, and the matrices $B, A_{0}, B_{0}, C$, $D, C_{0}, D_{0}, F_{2}, G$ are zero.

For such a system, let's consider the problem b, i.e. we find the control $u_{20}$ (3.2), that ensures the asymptotic stability of the solution $z=\varphi=0$ with respect to the variables $z, \rho$ with the smallest value of the quantity $\rho_{u}=u_{2}^{2}$. An isochoric process takes place in the heating furnace, in which the furnace pressure is proportional to the temperature, i.e.

$$
\varphi_{1}(1, t)=k_{2} z
$$

where $k_{2}>0$ - the constant gain.

In this example, the matrices $P_{1}$ and $P_{2}$ are absent. By equations (2.2), (2.3), (3.3) taking into account (4.4) the matrices $v(x), Q, w, \omega$ are developed with the following elements:

$$
v_{11}=\frac{1+\lambda}{\sqrt{b d \lambda(2+\lambda)}}, \quad v_{22}=\frac{b}{d} v_{11}, \quad v_{12}=\frac{1}{d}(1-x), \quad q=\frac{\theta b k_{2}}{k_{1}} v_{11}, \quad w_{11}=1,
$$




$$
w_{22}=0, \quad \omega=\frac{2 q}{\theta} .
$$

The conditions (2.6) on stability are satisfied. Therefore, the control $u_{20}(3.2)$, which in this case will be written in the following form:

$$
u_{20}=-\sqrt{\frac{b \lambda}{d(\lambda+2)}} \varphi_{2}(0, t),
$$

Is the solution to the problem $b$ for the system (3.1) - (3.3).

\section{Conclusions}

- Using the method of Lyapunov functions, a set of controls is determined that ensure the asymptotic stability of the considered system with distributed and lumped parameters.

- On this set, using the Lagrange multiplier method, optimal control is developed with the smallest norm value at each moment of time.

- The work has theoretical and practical value.

- The developed control laws are lumped controls applied to finite-dimensional links and (or) to the boundaries of the distributed link and can be implemented quite simply and accurately in practice.

\section{Summary}

When they solve the problems of control synthesis in the systems with distributed and lumped parameters, described by linear equations in partial and ordinary derivatives, a new approach is being developed related to the idea of the original higher-order equation transformation into a system of first-order equations by time and spatial coordinates. The transition to the first-order equations allows to develop the Lyapunov function constructively in the form of a sum of integral and ordinary quadratic forms, to obtain simpler control laws as the functions of phase coordinates, and ensure the stability of a closed system. The results of the work expand the class of engineering objects for which the control synthesis problem can be solved successfully.

\section{Acknowledgements}

The work is performed according to the Russian Government Program of Competitive Growth of Kazan Federal University.

\section{References}

[1] Bairamov, F.D., \& Bairamov, B.F. (2018). About Hybrid System Stability with Distributed and Concentrated Parameters. HELIX, 8(1), 2493-2498.

[2] Bairamov, F.D., \& Safronov, M.Yu. (2002). The Stability of systems with distributed parameters and lumped forces. J. Appl. Maths Mechs, 66(3), 341-345.

[3] Bayramov, B.F., \& Bayramov, F.D. (2018). On the Stability of one class of linear systems with distributed and lumped parameters. Applied Mathematics and Mechanics, Moscow, 82(6), 757-763.

[4] Bayramov, F.D. (1995), stability and optimal stabilization of the systems with distributed parameters. Moscow: Mashinostroenie, $160 \mathrm{p}$.

[5] Bayramov, F.D., Bayramov, B.F., Galimov, N.S., \& Fardeev, A.R. (2016). Technological processes with the combined power supply: mechanics, control, automation. Kazan: Publishing house of Kazan University, 342 p.

[6] Malkin, I.G. (1952). The theory of motion stability. Moscow: Gostekhizdat, $432 \mathrm{p}$.

[7] Parks, P.C. (1967). A stability criterion for a panel flutter problem via the second method of Liapunov. Differential Equations and Dynamical Systems, N.Y.; L.: Academic Press: 287-298.

[8] Sirazetdinov, T.K. (1987). Stability of the systems with distributed parameters. Novosibirsk: Nauka. Siberian branch, $231 \mathrm{p}$.

[9] Wang, P.K.C. (1966). Stability analysis of elastic and aeroelastic systems via Liapunov's direct method. Jorn. Frank. Inst., 281(1), 218-231.

[10] Wang, P.K.C. (1968). Theory of Stability and control for distributed parameter systems. Int. J. Control, 7(2), 101-116 (Bibliography). 